\title{
THE IDENTIFICATION OF NONDOMINATED AND EFFICIENT PATHS ON A NETWORK
}

G. R. Jahanshahloo ${ }^{\text {a }}$, F. Hossienzadeh Lotfi ${ }^{\text {a }}$, N. Shoja ${ }^{\text {b }}$, G. Tohidi ${ }^{c}$, S. Razavyan ${ }^{d}$

${ }^{a}$ Department of Mathematics, Science \& Research Branch, Islamic Azad University, Tehran-Iran

${ }^{\mathrm{b}}$ Department of Mathematics, Islamic Azad University, Firuozkooh Branch, Firuozkooh-Iran

${ }^{c}$ Department of Mathematics, Islamic Azad University, Tehran-Center Branch, Tehran-Iran, P.O. Box: 14515-459, ghtohidi@yahoo.com

${ }^{\mathrm{d}}$ Department of Mathematics, Islamic Azad University, Tehran-South Branch, Tehran-Iran

\begin{abstract}
In this paper, we present an application of 0-1 linear programming problem in the indentification of the nondominated paths on a network. To find efficient paths, an adaptationof the Additive model, which called Additive model without output, is used.

Key Words- Data Envelopment Analysis, 0-1 Linear Programming, Efficiency
\end{abstract}

\section{INTRODUCTION}

Data Envelopment Analysis (DEA) originating from Farell's [9] seminal work and popularized by Charnes et al. [6], provides a flexible nonparametric doctrine for empirical production analysis. In recent decades, DEA has rapidly expanded towards new application areas (see e.g. Seiford [11] for survey). The technique aroused great interest as the development of several variant of the CCR model [6] and their applications demonstrate. There are many alternative DEA models with different characteristics such as the BCC model [2], the Multiplicative models [7], the Additive model [4] and the FDH model [14,8]. Since the choice of a particular DEA model determines consequences on the study, careful considerations shoulde precede the selection of the model to solve any evaluation problem. Overviews on the subject are presented in Refs. [13,3,1] and some applications are cited in Ref. [12]. Other important references on DEA include some books such as $[5,10]$.

This paper deals with a technique based on 0-1 Linear Programming problem that identifies nondominated paths on a network. Then, these paths are evaluated by an adaptation of the Additive model. The rest of the paper unfolds as follows. Section 2, presents a method for determining of nondominated paths. In section 3 , we specify the efficient paths among nondominated paths. Finally, section 4 draws our conclusive remarks.

\section{APPOINTMENT OF NONDOMINATED PATHS}

Suppose we have $n$ paths in a network characterized by $m$ costs on each arc, the costs are independent and noncommensurate. Each of them represents a factor that characterizes paths quality. We denote the costs vectors related to path $j$ as $X_{j}=\left(x_{1 j}, \ldots, x_{m j}\right), j=1, \ldots, n$. Let $S=\left\{X_{j} \mid j=1, \ldots, n\right\}$ be a set of m-dimensional vectors related to all paths. 
Definition 2.1. We say that $X_{k}=\left(x_{1 k}, \ldots, x_{m k}\right)$ is nondominated in $S$ if and only if there is not $X_{l} \in S$ such that, $X_{k} \geq X_{l}$ with at least one strict inequality. Otherwise, we say that $X_{l}$ dominates $X_{k}$ in $S$.

Definition 2.2. The vector $\bar{X}=\left(\bar{x}_{1}, \ldots, \bar{x}_{m}\right)$ where

$$
\bar{x}_{i}=\min _{1 \leq j \leq n}\left\{x_{i j}\right\}, \quad i=1, \ldots, m
$$

is called the ideal of paths.

Theorem 2.1. If there are $i$ and $k$ such that $k$ is unique and $x_{i k}=\bar{x}_{i}$ then $k^{\text {th }}$ path is nondominated.

Proof: Since $\bar{x}_{i}=x_{i k}=\min _{1 \leq j \leq n}\left\{x_{i j}\right\}$ and $x_{i k}$ unique,so

$$
\bar{x}_{i}=x_{i k}<x_{i j}, \quad j=1, \ldots, n, j \neq k .
$$

Therefore, there is not $X_{j} \in S(j \neq k)$ so that $X_{j} \leq X_{k}$ and $X_{j} \neq X_{k}$. Consequently, based on definition $2.1, k^{\text {th }}$ path is nondominated. $\square$

Suppose that $S_{o}=\left\{X_{i_{1}}, \ldots, X_{i_{k}}\right\}$ is the set of vectors corresponding to the paths which have been distinguished nondominated by using Theorem 2.1. The distance of each vector $X \in S_{o}^{\prime}=S-S_{o}$ from $\bar{X}$ by using $l_{l}-$ norm is as $L(X, \bar{X})=\sum_{i=1}^{m}\left|\bar{x}_{i}-x_{i}\right|$. Since $\bar{x}_{i} \leq x_{i}(i=1, \ldots, m)$ so, $L(X, \bar{X})=\sum_{i=1}^{m}\left(x_{i}-\bar{x}_{i}\right)=\sum_{i=1}^{m} x_{i}-\sum_{i=1}^{m} \bar{x}_{i}$.

To find other nondominated paths, we specify a path from $S_{o}^{\prime}$ that is dominated by none members of $S_{o}$ and it has the shortest distance from ideal. To do so, consider the following problem in which $\alpha=-\sum_{i=1}^{m} \bar{x}_{i}$ is constant.

$\operatorname{Min} \quad L(X, \bar{X})=\sum_{i=1}^{m} x_{i}+\alpha$

$$
\begin{aligned}
& \text { s.t } \quad x_{i p}>\sum_{j \in S_{o}^{\prime}} t_{j} x_{i j}-M w_{i p}, \quad i=1, \ldots, m, \quad p \in S_{o} \\
& \sum_{i=1}^{m} w_{i p} \leq m-1, \quad p \in S_{o} \\
& \sum_{j \in S_{o}^{\prime}} t_{j}=1 \\
& w_{i p}, t_{j} \in\{0,1\}, \quad i=1, \ldots, m, P \in S_{o}, j \in S_{o}^{\prime}
\end{aligned}
$$

where $M$ is a positive large number and constraints $\sum_{i=1}^{m} w_{i p} \leq m-1,\left(p \in S_{o}\right)$ imply that for each $p \in S_{o}$, at least one of constraints be active and other constraints are redundant. Since $t_{j} \in\{0,1\}$ and $\sum_{j \in S_{o}^{\prime}} t_{j}=1$, the vectore $\sum_{j \in S_{o}^{\prime}} t_{j} X_{j}$ is one of the members $S_{o}^{\prime}$. Therefore, the model (2) is converted in the following model: 


$$
\begin{aligned}
& \operatorname{Min} \sum_{j \in S_{o}^{\prime}} \sum_{i=1}^{m} x_{i j} t_{j} \\
& \text { s.t } \quad x_{i p}>\sum_{j \in S_{o}^{\prime}} t_{j} x_{i j}-M w_{i p}, \quad i=1, \ldots, m, p \in S_{o} \\
& \sum_{i=1}^{m} w_{i p} \leq m-1, \quad p \in S_{o} \\
& \sum_{j \in S_{o}^{\prime}} t_{j}=1 \\
& w_{i p}, t_{j} \in\{0,1\}, \quad i=1, \ldots, m, p \in S_{o}, j \in S_{o}^{\prime} .
\end{aligned}
$$

Theorem 2.2. If in optimal solution of problem (3) $t_{h}^{*}=1$, then $X_{h} \in S_{o}^{\prime}$ is nondominated.

Proof: We show that $X_{h}$ is dominated by none of members $S_{o}$ and $S_{o}^{\prime}$. Assume that $\left(T^{*}, W^{*}\right)$ is an optimal solution of the problem (3) with components $t_{j}^{*}\left(j \in s_{o}^{\prime}\right)$ and $w_{i p}^{*}\left(p \in s_{o}, i=1, \ldots, m\right)$. Since $\sum_{i=1}^{m} w_{i p}^{*} \leq m-1$, there is at least one index $i$ which $w_{i p}^{*}=0$. Hence, for each $p \in S_{o}$ we have $x_{i p}>x_{i h}$. Consequently, $p^{\text {th }}$ path does not dominate $h^{\text {th }}$ path. By contradiction, suppose that $X_{h} \in S_{o}^{\prime}$ dominates $X_{h}$ that is $X_{k} \leq X_{h}$ and $X_{k} \neq X_{h}$. Therefore

$$
x_{i k} \leq x_{i h}, \quad i=1, \ldots, m
$$

and at least one of inequalities (4) strictly hold. By summing the inequalities (4), we will have, $\sum_{i=1}^{m} x_{i k}<\sum_{i=1}^{m} x_{i h}$. This shows $L\left(X_{k}, \bar{X}\right)<L\left(X_{h}, \bar{X}\right)$. Since $X_{h}$ is dominated by none of members $S_{o}$, any member of $S_{o}$ will not dominate $X_{k}$. Therefore $\left(T^{*}, W^{*}\right)$ will not be optimal solution of the problem (3), which is a contradiction.

In order to develop the algorithm for finding nondominated paths, the sets $S_{k}$ and $S_{k}^{\prime}$ are defined as follows:

$S_{k}: S_{k}$ is a subset of $S$ that denotes the set of paths which has been specified nondominated until $k^{\text {th }}$ iteration.

$S_{k}^{\prime}: S_{k}^{\prime}=S-S_{k}$.

\section{An Algorithm for Appointment of Nondominated Paths \\ Stage 0: Initialization}

Step 0: Identify the members $S_{o}$ by using (1),

\section{Stage 1: Identification}

Step 1-1: Solve the problem (3),

Step 1-2: If there is not $h$ where $t_{h}=1$, stop. Otherwise, put $S_{k+1}=S_{k} \cup\left\{X_{h}\right\}$ and go to step 1-1,

Stage 2: End. 


\section{DETERMINIG EFFCIENT PATHS}

Suppose that we have $n$ DMUs with input vectors $X_{j}(j=1, \ldots, n)$ and output vectors $Y_{j}(j=1, \ldots, n)$. To identify the efficient DMUs, we can apply the Additive model [5] which is as follows:

$$
\begin{aligned}
& P_{o}^{*}=\operatorname{Max} \sum_{i=1}^{m} s_{i}^{-}+\sum_{r=1}^{s} s_{r}^{+} \\
& \text {s.t. } \quad \sum_{j=1}^{n} \lambda_{j} y_{r j}-s_{r}^{+}=y_{r o}, \quad r=1, \ldots, S \\
& \sum_{j=1}^{n} \lambda_{j} x_{i j}+s_{i}^{-}=x_{i o}, \quad i=1, \ldots, m \\
& \sum_{j=1}^{n} \lambda_{j}=1 \\
& \lambda_{j} \geq 0, s_{i}^{-} \geq 0, s_{r}^{+} \geq 0, \quad j=1, \ldots, n, \quad i=1, \ldots, m, \quad r=1, \ldots, s .
\end{aligned}
$$

It has been proved that a DMU is efficient if and only if $P_{o}^{*}=0$ in the model (5). An interesting property of Additive model is the translation invariance proved by Ali and Seiford [1]. Feasibility And boundedness are the other properties of this model.

Using DEA terminology, we observe that the paths in the network are units to compare, each of then is described the set of the costs (inputs). In DEA, this units are considered as DMUs without output.

Definition 3.1. A unit $k$ is efficient if and only if there is not a convex combination of the units such that every component of the convex combination is less than or equal $X_{k}$ and at least one component is not equal.

Since the units corresponding to the paths in the network have not the output vectors, we can evaluate these units by the following model which is called the Additive model without outputs (envelopment form).

$$
\begin{aligned}
P_{o}^{* *}=\operatorname{Max} & \sum_{i=1}^{m} s_{i}^{-} \\
\text {s.t. } & \sum_{j=1}^{n} \lambda_{j} x_{i j}+s_{i}^{-}=x_{i o}, \quad i=1, \ldots, m \\
& \sum_{j=1}^{n} \lambda_{j}=1 \\
& \lambda_{j} \geq 0, s_{i}^{-} \geq 0, \quad j=1, \ldots, n, \quad i=1, \ldots, m .
\end{aligned}
$$

It is evident that if $P_{o}^{\prime *}=0$ then, unit $o$ is surely efficient, otherwise it is inefficient.

Theorem 3.1. The Additive model without output is feasible and bounded.

Proof: $\lambda_{o}=1, \lambda_{j}=0,(j \neq o), s_{i}^{-}=0,(i=1, \ldots, m)$ is a feasible solution of problem (6). Consider the dual form (multiplier form) of the model (6) which is as follows: 


$$
\begin{array}{rlr}
D_{o}^{* *}=\operatorname{Min} & \sum_{i=1}^{m} v_{i o} x_{i o}+u_{o} & \\
\text { s.t. } & \sum_{j=1}^{n} v_{i o} x_{i j}+u_{o} \geq 0, & j=1, \ldots, n \\
& v_{i o} \geq 1, & i=1, \ldots, m .
\end{array}
$$

Since $\left(v_{1 o}, \ldots, v_{m o}, u_{o}\right)=\left(1, \ldots, 1, u_{o}\right)$ is feasible solution for $(7)$, where $u_{o}=\max _{1 \leq j \leq n}\left\{\sum_{i=1}^{m} x_{i j}\right\}$, therefore the model (6) is bounded.

Theorem 3.2. The Additive model without output is translation invariance.

The proof is straightforward.

We know that each efficient unit(path) is a nondominated path. Hence, if $K=\left\{X_{i_{1}}, \ldots, X_{i_{l}}\right\}$ be the set of vectors corresponding to the paths which have been distinguished nondominated by means of algorithm of the section 2 , then the efficient paths are determined by evaluating the members of $K$ by model (5).

Note that the efficient paths can be determined by evaluating units corresponding to all the paths by model (6).

\section{EXAMPLE}

Suppose there exist eight paths which each path has two attributes (the costs). The costs of these paths have been reported in Table 1.

Table 1: the costs of the paths

\begin{tabular}{|lllllllll|}
\hline No. & 1 & 2 & 3 & 4 & 5 & 6 & 7 & 8 \\
\hline Attribute1 & 124 & 263 & 82 & 338 & 434 & 140 & 130 & 438 \\
\hline Attribute2 & 25 & 26 & 26 & 35 & 53 & 14 & 34 & 50 \\
\hline
\end{tabular}

We first identify the nondominated paths by using the presented algorithm.

Step 0: We have $\bar{x}_{1}=\min _{1 \leq j \leq 8}\left\{x_{1 j}\right\}=82 \quad$ and $\quad \bar{x}_{2}=\min _{1 \leq j \leq 8}\left\{x_{2 j}\right\}=14$. Hence, $S_{o}=\left\{X_{3}, X_{6}\right\}=\{(82,26),(140,14)\}$.

\section{Iteration 1 - Stage 1:}

Step 1-1: In this step, we solve the following problem:

$$
\begin{array}{lll}
\text { Min } & 149 t_{1}+289 t_{2}+373 t_{4}+487 t_{5}+164 t_{7}+484 t_{8} & \\
\text { S.t } \quad & 124 t_{1}+263 t_{2}+338 t_{4}+434 t_{5}+130 t_{7}+434 t_{8}-1000 w_{13}<82 \\
& 25 t_{1}+26 t_{2}+35 t_{4}+53 t_{5}+34 t_{7}+50 t_{8}-1000 w_{23} & <26 \\
& 124 t_{1}+263 t_{2}+338 t_{4}+434 t_{5}+130 t_{7}+434 t_{8}-1000 w_{16}<140 \\
& 25 t_{1}+26 t_{2}+35 t_{4}+53 t_{5}+34 t_{7}+50 t_{8}-1000 w_{26} & <14 \\
& t_{1}+t_{2}+t_{4}+t_{5}+t_{7}+t_{8}=1 \\
& w_{13}+w_{23} \leq 1 \\
& w_{16}+w_{26} \leq 1 \\
& t_{1}, t_{2}, t_{4}, t_{5}, t_{7}, t_{8}, w_{13}, w_{23}, w_{16}, w_{26} \in\{0,1\} .
\end{array}
$$


The optimal solution of the above problem is as follows:

$$
\left(t_{1}^{*}, t_{2}^{*}, t_{4}^{*}, t_{5}^{*}, t_{7}^{*}, t_{8}^{*}, w_{13}^{*}, w_{23}^{*}, w_{16}^{*}, w_{26}^{*}\right)=(1,0,0,0,0,0,1,0,0,1)
$$

Step 1-2: Since $t_{1}^{*}=1$, so $S_{1}=\left\{X_{1}, X_{3}, X_{6}\right\}=\{(124,25),(106,33),(140,14)\}$.

\section{Iteration 2 - Stage 1:}

Step 1-1: In this step, we solve the following problem:

$$
\begin{array}{lll}
\text { Min } & 289 t_{2}+373 t_{4}+487 t_{5}+164 t_{7}+484 t_{8} & \\
\text { S.t } & 263 t_{2}+338 t_{4}+434 t_{5}+130 t_{7}+434 t_{8}-1000 w_{13} & <82 \\
& 26 t_{2}+35 t_{4}+53 t_{5}+34 t_{7}+50 t_{8}-1000 w_{23} & <26 \\
& 263 t_{2}+338 t_{4}+434 t_{5}+130 t_{7}+434 t_{8}-1000 w_{16} & <140 \\
& 26 t_{2}+35 t_{4}+53 t_{5}+34 t_{7}+50 t_{8}-1000 w_{26} & <14 \\
& 263 t_{2}+338 t_{4}+434 t_{5}+130 t_{7}+434 t_{8}-1000 w_{16} & <124 \\
& 26 t_{2}+35 t_{4}+53 t_{5}+34 t_{7}+50 t_{8}-1000 w_{26} & <14 \\
& t_{2}+t_{4}+t_{5}+t_{7}+t_{8}=1 & \\
& w_{13}+w_{21} \leq 1 & \\
& w_{13}+w_{23} \leq 1 & \\
& w_{16}+w_{26} \leq 1 & \\
& t_{2}, t_{4}, t_{5}, t_{7}, t_{8}, w_{11}, w_{21}, w_{13}, w_{23}, w_{16}, w_{26} \in\{0,1\} .
\end{array}
$$
follows:

The above problem is infeasible. Therefore, the set of nondominated paths is as

$$
S_{1}=\left\{X_{1}, X_{3}, X_{6}\right\}=\{(124,25),(106,33),(140,14)\} .
$$

In order to determine the efficient paths among nondominated paths, we evaluate the corresponding units of the members $S_{1}$ by model (6). The results of evaluation has been reported in Table 2 .

Table 2: the results of evaluation

\begin{tabular}{|lllllll|}
\hline & $P_{o}^{\text {'* }}$ & $\lambda_{1}^{*}$ & $\lambda_{3}^{*}$ & $\lambda_{6}^{*}$ & $s_{1}^{-*}$ & $s_{2}^{-*}$ \\
\hline$X_{1}$ & 3.6842 & 0 & 0.5789 & 0.4211 & 3.6842 & 0 \\
\hline$X_{3}$ & 0 & 0 & 1 & 0 & 0 & 0 \\
\hline$X_{6}$ & 0 & 0 & 0 & 1 & 0 & 0 \\
\hline
\end{tabular}

Table 2 shows that the paths $X_{3}$ and $X_{6}$ are efficient but the path $X_{1}$ is not efficient.

\section{CONCLUSION}

This paper presents a method for determining nondominated paths by using 0-1 linear programing problem. To construct 0-1 linear programing problem has been used from $l_{1}$ norm and concept of nondominance. The efficient paths are specified by evaluating nondominated paths or all paths by means of the Additive model without output. 


\section{REFERENCES}

1. A. I. Ali, C. S. Lerme and L. M. Seiford, Components of efficiency evaluation in DEA, European Journal of Operational Research 80, 462-473, 1995.

2. R. D. Banker, A. Charnes and W.W. Cooper, Some method for estimating technical and scale inefficiencies in data envelopment analysis, Management Science 30 (9), 1078-1092, 1984.

3. A. Boussofiane, R. G. Dyson and E. Thanassoulis, Applied DEA, European Journal of Operational Research 52, 1-15, 1984.

4. A. Charnes, W.W. Cooper, B. Golany, L. M. Seiford and J. Stutz, Foundation of DEA for pareto koopmans efficient empirical production functions, Journal of Econometrics, 30 (12), 91-107, 1985.

5. A. Charnes, W.W. Cooper, A. Y. Lewin and L. M. Seiford, DEA: Theory, methodology and applications, Kluwer Academic Publisher Norwell, MA, 1994.

6. A. Charnes, W.W. Cooper and E. Rhodes, Measuring the efficiency of decision making units, European Journal of Operational Research 2(6), 429-444, 1978.

7. A. Charnes, W.W. Cooper, L. M. Seiford and J. Stutz, A multiplicative model for efficiency analysis, Socio-Economic Planning Sciences 16 (5), 223-224, 1982.

8. P. H. Eeckaut and H. Tulkens, Nonparametric efficiency, progress and regress measures for panel data: methodological aspects, European Journal of Operational Research 80, 474-499, 1995.

9. M. J. Farrell, The measurement of productive efficiency, Journal of Royal Statistical Society, Series A 120(3), 253-290, 1957.

10. H. Fried, K. Lovell and S. Schmidt, The measurement of productive efficiency: techniques and applications, Oxford University Press New York, 1993.

11. L. M. Seiford, Data envelopment analysis: the evolution of the state of the art, Journal of Productivity Analysis, 7, 99-137, 1996.

12. L. M. Seiford, A bibliography of DEA, Working Paper, Department of industrial engineering and Operations Research, The University of Massachusetts, MA, 1990.

13. L. M. Seiford, and R. M. Thrall, Recent developments in DEA, Journal of Econometrics, 46, 7-38, 1990.

14. H. Tulkens, On FDH analysis: some methodological issues and applications to retail banking, courts and urban transit, Journal of Productivity Analysis, 4 (1), 183-210, 1993. 